\title{
Long-Term Follow-up from the Treatment and Crossover Arms of a Randomized Controlled Trial of an Absorbable Nasal Implant for Dynamic Nasal Valve Collapse
}

\author{
Nadim Bikhazi, MD ${ }^{1}$ Randall A. Ow, MD² Ellen M. O'Malley, MS ${ }^{3}$ (1) Nora Perkins, MD ${ }^{4}$ \\ Douglas M. Sidle, MD ${ }^{5}$ Pablo Stolovitzky, MD ${ }^{6}$
}

${ }^{1}$ Department of ENT, Ogden Clinic, South Ogden, Utah

2 Sacramento Ear Nose and Throat Medical and Surgical Group, Roseville, California

${ }^{3}$ ENT Clinical Affairs, Stryker Corp, Plymouth, Minnesota

${ }^{4}$ Department of ENT, Albany ENT and Allergy Services, Albany, New York

${ }^{5}$ Department of Otolarygology-Head and Neck Surgery,

Northwestern University Feinberg School of Medicine, Chicago, Illinois

${ }^{6}$ Department of Otolaryngology, Emory University School of

Medicine, Atlanta, Georgia

Facial Plast Surg 2022;38:495-503.

\author{
Address for correspondence Ellen M. O'Malley, MS, ENT Clinical Affairs, \\ Stryker Corp, 3600 Holly Lane North, Suite 40, Plymouth, MN 55447 \\ (e-mail: ellen.omalley@stryker.com; marilyn.dysart@stryker.com).
}

\begin{abstract}
Keywords

- nasal valve collapse

- nasal airway obstruction

- bioabsorbable implant

- lateral wall

- nasal valve collapse

We report the long-term safety and efficacy outcomes of the treatment and crossover arms of a randomized controlled trial evaluating an absorbable nasal implant to address dynamic nasal valve collapse. Participants were adults with severe/extreme nasal airway obstruction primarily due to nasal valve insufficiency who had implant placement. Follow-up visits were at 3, 6, 12, 18, and 24 months post implant. Visits included collection of the following patient-reported outcome measures: nasal obstructive symptom evaluation (NOSE), nasal obstruction visual analog scale (VAS), and the Epworth Sleepiness Scale (ESS). Adverse events were evaluated at each visit. One-hundred-eleven participants with implants were followed. Of the 111, 90 completed the 12-month visit and 70 completed the 24-month visit. NOSE responder rates are greater than $80 \%$ at all follow-ups through 24 months. Mean reduction from baseline in NOSE scores is $\geq 30$ points and statistically significant $(p<0.001)$ at all time points through 24 months. Mean VAS score reduction is $\geq 29.7$ points and statistically significant $(p<0.001)$ at all time points. The subgroup of participants with baseline ESS values $>10$ experienced statistically significant $(p<0.001)$ and clinically meaningful reductions at all postimplant periods, suggesting that the reduction in nasal symptoms may reduce daytime sleepiness for patients who have problems with sleep quality. No serious device-/procedure-related adverse events were reported. Implant migration/retrieval rate was $4.5 \%$ (10/222) of total implants or $9 \%$ of participants (10/111). The implant is safe and effective for dynamic nasal valve collapse in patients with severe/extreme nasal obstruction and provides durable symptom improvement 24 months after placement.
\end{abstract}

Issue Theme Meaningful Innovations in Rhinoplasty; Guest Editor: Peter William Hellings, MD, PhD

DOI https://doi.org/ 10.1055/s-0041-1740948. ISSN $0736-6825$. (c) 2022. The Author(s).

This is an open access article published by Thieme under the terms of the Creative Commons Attribution-NonDerivative-NonCommercial-License, permitting copying and reproduction so long as the original work is given appropriate credit. Contents may not be used for commercial purposes, or adapted, remixed, transformed or built upon. (https://creativecommons.org/ licenses/by-nc-nd/4.0/)

Thieme Medical Publishers, Inc., 333 Seventh Avenue, 18th Floor, New York, NY 10001, USA 
More than 20 million Americans ${ }^{1}$ are estimated to suffer from nasal airway obstruction, which limits airflow through the nose with significant quality of life consequences. ${ }^{2}$ Even slight narrowing of the nasal valve can lead to significant reduction in airflow. ${ }^{3,4}$ Symptoms may include nasal congestion or stuffiness, nasal blockage or obstruction, trouble sleeping, and inability to get enough air through the nose during exercise or exertion. Structural blockages typically occur at the septum, turbinates, and/or lateral nasal wall. Therefore, the most common conditions that impact patients with nasal airway obstruction are septal deviation, turbinate hypertrophy, and nasal valve collapse (NVC). Lateral wall collapse may equal or even exceed septal deviation as the prime cause of nasal airway obstruction. ${ }^{5-8}$ NVC has been found to be present in up to $82 \%$ of patients who have had previous septoplasty and/or turbinate reduction, suggesting it is overlooked as a causative factor of nasal obstruction. ${ }^{8}$

Nonsurgical therapies such as Breathe Right strips or nasal cones have been reported to offer temporary relief, but do not address the root cause of the problem. Alternatively, surgical treatments including septoplasty or inferior turbinate reduction may alleviate impaired nasal breathing in patients; however, these procedures still do not directly address the weakened lateral wall. ${ }^{5,9}$ Surgery to strengthen the lateral wall has been shown to significantly improve the quality of life for patients suffering from nasal airway obstruction $^{10}$; however, the procedures are invasive, with risk of collapse of the alar rim or lateral crura, graft resorption, scarring, and graft necrosis. Furthermore, these procedures typically require general anesthesia, which not only increases the risk of adverse events for the patient, but also significantly increases the complexity, cost, and time associated with the procedure. Tissue for cartilaginous grafts is typically harvested from the nasal septum, ribs, or ear cartilage $^{11,12}$ and placed as lateral crural strut grafts, alar batten grafts, splay grafts, rim grafts, or butterfly grafts. ${ }^{13-16}$

In the last several years, an absorbable implant made of a 70:30 copolymer of poly(L-lactide) and poly(D-lactide) has been developed that can be inserted under local anesthesia, using a minimally invasive technique. A recent randomized controlled trial demonstrated the superiority of the implant over a sham procedure. ${ }^{17}$ Here, we report the long-term safety and efficacy outcomes of the combined treatment and crossover arms of the randomized controlled trial evaluating the absorbable nasal implant.

\section{Materials and Methods}

\section{Study Design and Population}

This study is a prospective, multicenter, randomized, shamcontrolled, crossover postmarket trial of the LATERA absorbable nasal implant (Stryker, Plymouth, MN 55447). The study design, methods, and 3-month results of the comparison between the treatment and the sham arms were previously reported. ${ }^{17}$ Briefly, the aim of this trial was to compare the outcomes for patients with severe to extreme Nasal Obstruction Symptom Evaluation (NOSE) scores treated with the 70:30 poly (L-lactide) and poly (D-lactide) LATERA bioab- sorbable nasal implant with those treated with a sham control procedure. Here we report the results of the treatment and crossover arms through 24-month follow-up.

The study was reviewed and approved by Advarra IRB, Columbia, Maryland. All participants provided written informed consent before participating in the study and were enrolled at the time of consent. Upon enrollment, baseline data were collected including demographic information, general medical history, nasal medical history including risk factors, NAO breathing assessment using a visual analog scale (VAS), NOSE score, nasal exam including assessment of the septum and turbinates, modified Cottle maneuver, and pregnancy test for women of childbearing potential. The study is registered and results are posted at www.clinicaltrials.gov (NCT 03400787).

Participants were adults with severe to extreme nasal airway obstruction that was primarily due to nasal valve insufficiency. Inclusion criteria included a baseline Nasal Obstruction Symptom Evaluation (NOSE) ${ }^{18}$ score of 55 or greater and a positive modified Cottle maneuver. Additionally, participants were required to have documentation of lack of benefit or tolerability of at least 4 weeks of conservative medical management (e.g., antihistamines or nasal steroids). Participants were excluded from enrollment if they required concurrent nasal procedures or had undergone endoscopic sinus surgery, septoplasty, inferior turbinate reduction, or rhinoplasty within 6 months before enrollment. External nasal dilators were not permitted during the study.

Initially, participants were randomized (1:1) to treatment or a sham procedure. Procedures were performed in the physician's office, where participants in the active treatment arm received the LATERA implant delivered using a cannula inserted into the nasal wall and participants in the sham control had an identical cannula inserted into the nasal lateral wall but received no implant. - Fig. 1 demonstrates the insertion and optimal placement of the LATERA implant
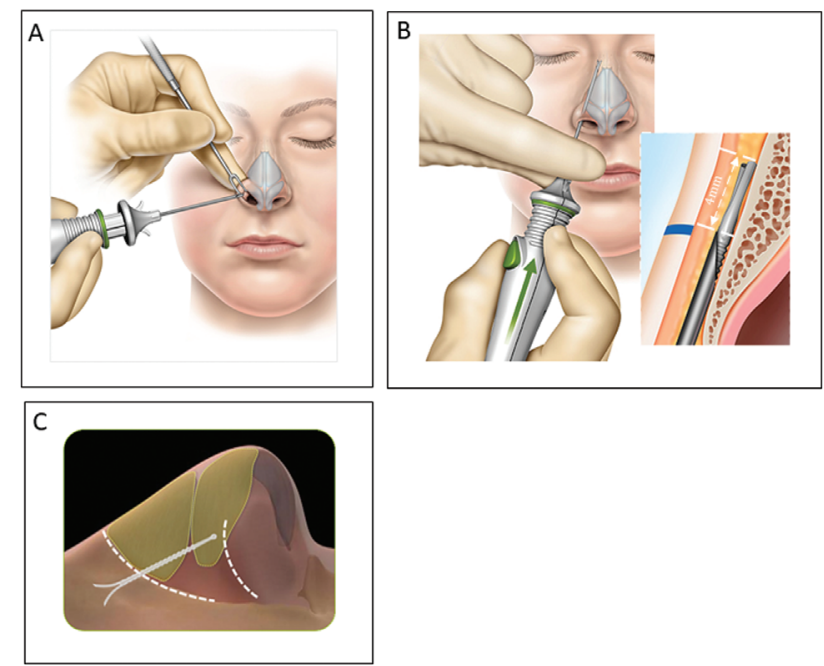

Fig. 1 LATERA implant delivery process and optimal placement. (A) Delivery device orientation and (B) actuation of the delivery device with implant forks expanded approximately 4-mm distal to cannula tip resulting in (C) optimal implant location. 
in the lateral wall structure. All patients were followed for 3 months to evaluate the primary end point. After the 3month visit, sham participants still meeting inclusion criteria (i.e., NOSE score $\geq 55$ ) were invited to crossover to the treatment arm and were then followed for up to 24 months post placement.

\section{Assessments}

Participants underwent follow-up visits at 3, 6, 12, 18, and 24 months post implant. Visits included collection of patientreported outcome measures of the NOSE, a nasal obstruction visual analog scale (VAS), and the Epworth Sleepiness Scale (ESS). ${ }^{19}$ Adverse event reporting was also evaluated at each visit. A NOSE responder was defined as a participant with at least 1 NOSE class improvement or a NOSE score reduction of at least $20 \%$ compared with baseline. NOSE classes were as described by Lipan and Most. ${ }^{20}$ Partial NOSE responses were considered invalid.

The patient-reported nasal obstruction VAS was assessed at baseline and at each follow-up visit. The participants marked their current perception of nasal obstruction on a $100-\mathrm{mm}$ horizontal line with the $0-\mathrm{mm}$ end indicating "no difficulty/easy to breathe through the nose" and the 100-mm end indicating "unable to breathe through the nose." The score reported is the millimeter marking made by the participant on the scale.

The ESS was assessed at baseline and at all follow-up visits. The questionnaire evaluates a participant's tendency to become sleepy in eight common situations. Each situation is rated on a scale of 0 (no chance of dozing), to 3 (high chance of dozing). The responses are summed to provide a total score ranging from 0 to 24 . ESS scores greater than 10 are considered abnormal. $^{19}$

\section{Statistical Analysis}

Discrete variables are expressed as rates and proportions. Continuous variables are reported as means and standard deviations. For categorical variables, the numerator, denominator, and percentage are presented. For selected variables, the $95 \%$ confidence intervals $(\mathrm{CI})$ are presented. When presented, the 95\% CIs are calculated using the exact method. A two-sided binomial test of proportions is used to compare treatment groups for binary end points and a two-sample $t$-test is used for continuous end points.

To address the impact of the missing data at the 18-month and 24-month follow-ups on the NOSE outcomes, we conducted two ad hoc analyses. The first analysis uses the last observation carried forward (LOCF). In this analysis, the last NOSE score available from each discontinued participant is carried forward through the remaining follow-up periods. The second analysis is a worst-case scenario in which all missing NOSE values are set to baseline. The NOSE responder rate and mean change from baseline are calculated using these imputation methods.

The statistical analysis was conducted by an independent statistician and all statistical programming was performed using SAS version 9.4 or above.

\section{Results}

\section{Participants}

A total of 137 participants (71 treatment, 66 sham) were enrolled and treated in the randomized cohort. Twentythree sham participants did not qualify for crossover, one died and one withdrew before the 3-month follow-up, and one qualified but chose not to undergo the crossover procedure. The 40 remaining sham participants underwent a crossover procedure, resulting in 111 participants in the combined treatment and crossover arms for long-term follow-up. Of the 111, 90 completed the 12-month visit and 70 completed the 24-month visit. The reasons for early termination include 23 lost to follow-up, seven participants withdrew, four had lack of treatment effect and sought other treatments, and seven withdrew due to the COVID-19 pandemic. Most lost to follow-ups occurred after the 12-month visit, a time that overlapped with the COVID-19 pandemic. The flow of participants through the study is presented in -Fig. 2.

Demographics, baseline data, and medical history are presented by study arm in - Table $\mathbf{1}$. The baseline NOSE scores are slightly but significantly lower for the crossover arm compared with the treatment arm (76.1 \pm 13.3 vs. $69.4 \pm 14.5, p=0.015)$. This difference is due to the placebo effect observed in the sham group and reported in the initial paper. ${ }^{17}$ Otherwise, there are no significant differences between the arms at baseline. One participant was implanted on the left side only, all others received bilateral implants (one implant per side).

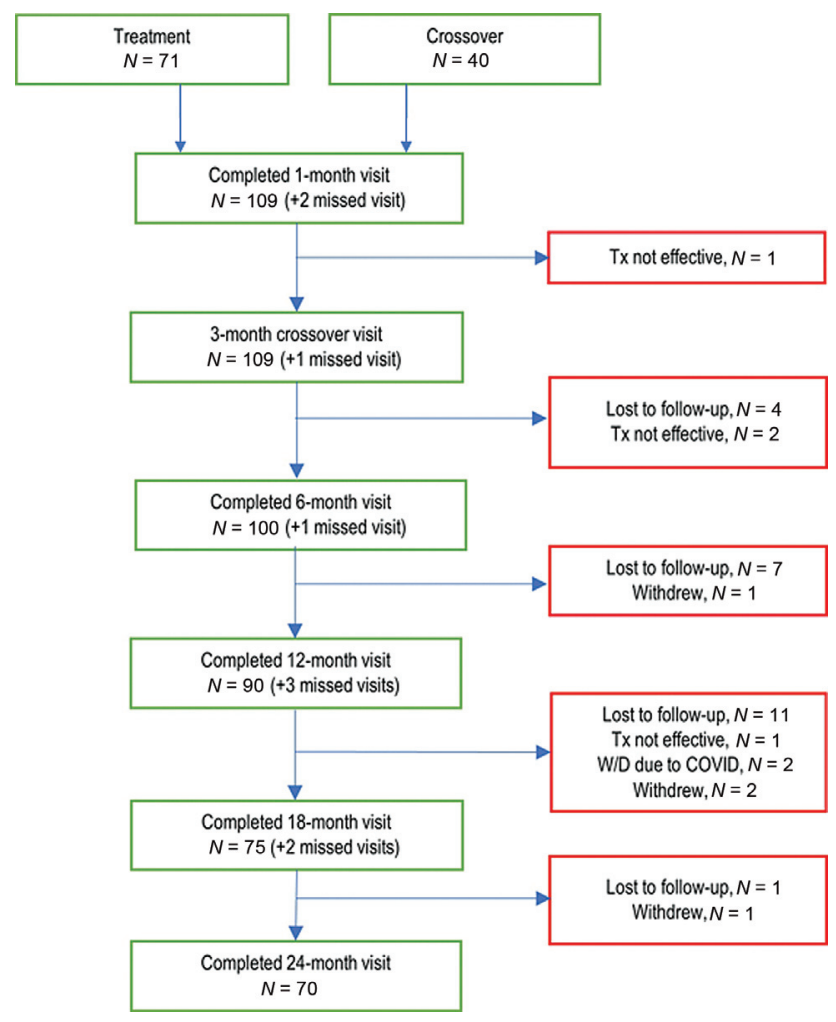

Fig. 2 Participant flow. 
Table 1 Demographics, baseline characteristics, and medical history

\begin{tabular}{|l|l|l|l|l|}
\hline Characteristic & Latera N=71 & CrossoverN=40 & $p$-Value ${ }^{\text {a }}$ & All participantsN=111 \\
\hline Age (years) & $51.5 \pm 13.7$ & $51.9 \pm 14.3$ & 0.909 & $51.6 \pm 13.9$ \\
\hline Sex (Male) & $40.8 \%(29)$ & $47.5 \%(40)$ & 0.552 & $43.2 \%(48)$ \\
\hline Body mass index $\left(\mathrm{kg} / \mathrm{m}^{2}\right)$ & $28.5 \pm 6.7$ & $29.2 \pm 5.4$ & 0.593 & $28.8 \pm 6.3$ \\
\hline Race & $2.8 \%(2)$ & $5.0 \%(2)$ & 0.618 & $3.6 \%(4)$ \\
\hline Asian & $1.4 \%(1)$ & $0.0 \%(0)$ & NA & $0.9 \%(1)$ \\
\hline American Indian/Alaskan Native & $0.0 \%(0)$ & $0.0 \%(0)$ & NA & $0.0 \%(0)$ \\
\hline Black or African American & $0.0 \%(0)$ & $0.0 \%(0)$ & NA & $0.0 \%(0)$ \\
\hline Native Hawaiian/Other Pacific Islander & $90.1 \%(64)$ & $90.0 \%(36)$ & 1.000 & $90.1 \%(100)$ \\
\hline White & $5.6 \%(4)$ & $5.0 \%(2)$ & 1.000 & $5.4 \%(6)$ \\
\hline Other ${ }^{\text {b }}$ & \multicolumn{5}{l|}{} \\
\hline Ethnicity & $12.7 \%(9)$ & $10.0 \%(4)$ & 0.767 & $11.7 \%(13)$ \\
\hline Hispanic/Latino & $85.9 \%(61)$ & $90.0 \%(36)$ & 0.767 & $87.4 \%(97)$ \\
\hline Not Hispanic/Latino & $0.0 \%(0)$ & $0.0 \%(0)$ & NA & $0.0 \%(0)$ \\
\hline Not reported & $0.0 \%(0)$ & $0.0 \%(0)$ & NA & $0.0 \%(0)$ \\
\hline Unknown & $76.1 \pm 13.3$ & $69.4 \pm 14.5$ & 0.015 & $73.6 \pm 14.0$ \\
\hline Baseline NOSE score & $75.7 \pm 13.3$ & $72.9 \pm 18.5$ & 0.406 & $74.7 \pm 15.3$ \\
\hline Baseline VAS score (nasal obstruction) & \multicolumn{5}{|l|}{} \\
\hline Medical history & $54.9 \%(39)$ & $72.5 \%(29)$ & 0.104 & $61.3 \%(68)$ \\
\hline Surgical history (nasal) & $38.0 \%(27)$ & $55.0 \%(22)$ & 0.111 & $44.1 \%(49)$ \\
\hline Allergic rhinitis & $19.7 \%(14)$ & $27.5 \%(11)$ & 0.355 & $22.5 \%(25)$ \\
\hline Sinus disease & $23.9 \%(17)$ & $27.5 \%(11)$ & 0.820 & $25.2 \%(28)$ \\
\hline Obstructive sleep apnea & $98.6 \%(69 / 70)$ & $95.0 \%(38)$ & 0.299 & $97.3 \%(107 / 110)$ \\
\hline Nonsurgical medical management & $88.2 \%(60 / 68)$ & $92.1 \%(35 / 38)$ & 0.743 & $89.6 \%(95 / 106)$ \\
\hline Mechanical nasal treatments &
\end{tabular}

Abbreviations: NOSE, Nasal Obstruction Symptom Evaluation; VAS, visual analog scale.

Note: Results are presented as mean \pm SD or \% ( $n$ ).

${ }^{a} p$-Values are based on two-sample $t$-test or Fishers exact test.

bOther races include: undetermined; Puerto Rican; mixed; Native American; and Hispanic.

\section{Patient-Reported Outcomes}

The NOSE responder rates are greater than $80 \%$ at all followups through 24 months (-Table 2 ). - Table 3 and - Fig. 3 show the mean reduction from baseline in NOSE scores to be

Table 2 NOSE responder rate

\begin{tabular}{|l|l|l|}
\hline Follow-up period & $n / N$ & Responder rate $(95 \% \mathrm{Cl})^{\mathrm{a}}$ \\
\hline $1 \mathrm{mo}$ & $85 / 104$ & $81.7 \%(72.9 \%, 88.6 \%)$ \\
\hline $3 \mathrm{mo}$ & $90 / 107$ & $84.1 \%(75.8 \%, 90.5 \%)$ \\
\hline $6 \mathrm{mo}$ & $88 / 100$ & $88.0 \%(80.0 \%, 93.6 \%)$ \\
\hline $12 \mathrm{mo}$ & $75 / 88$ & $85.2 \%(76.1 \%, 91.9 \%)$ \\
\hline $18 \mathrm{mo}$ & $66 / 74$ & $89.2 \%(79.8 \%, 95.2 \%)$ \\
\hline $24 \mathrm{mo}$ & $60 / 68$ & $88.2 \%(78.1 \%, 94.8 \%)$ \\
\hline
\end{tabular}

Abbreviations: $\mathrm{Cl}$, confidence interval; NOSE, Nasal Obstruction Symptom Evaluation.

${ }^{a}$ Responder is defined as a participant with at least 1 NOSE class improvement or a NOSE score reduction of at least $20 \%$ compared with baseline. Partial responses are considered invalid. greater than 30 points and statistically significant (all $p$ $<0.001$ ) at all time points through 24 months. The shift in NOSE class is shown in - Fig. 4 . At baseline, $98.2 \%$ of participants had either severe or extreme NOSE scores (protocol deviations were noted for inclusion criteria not met for two participants). At all follow-ups from 3 months through 24 months, $70.0 \%$ or more have improved to mild or moderate NOSE scores.

The LOCF analysis of the NOSE responder rate shows that the participant drop-off seen at the 18-month and 24-month visits does not impact the overall results. When carrying forward the last observation for participants who dropped out, the 18-month and 24-month responder rates are still both $87.4 \%(97 / 111 ; 95 \% \mathrm{CI} 79.7 \%, 92.9 \%)$ as compared with $89.2 \%$ at the 18 -month and $88.2 \%$ at the 24 -month visits for the participants with follow-up (-Table 2). The mean change in NOSE score is also not noticeably impacted with LOCFadjusted 18-month and 24-month mean changes of -38.7 and -37.7 , respectively, as compared with -39.8 and -38.4 in the participants with follow-up (-Table 3 ). 
Table 3 Change in mean NOSE scores at all follow-up periods

\begin{tabular}{|l|l|l|l|l|l|}
\hline Follow-up period & $N$ & Baseline NOSE & Visit NOSE & Mean change & $p_{\text {-Value }}{ }^{\mathbf{a}}$ \\
\hline $1 \mathrm{mo}$ & 104 & $74.1 \pm 13.9$ & $41.1 \pm 21.9$ & $-33.0 \pm 25.2$ & $<0.001$ \\
\hline $3 \mathrm{mo}$ & 107 & $73.8 \pm 13.9$ & $37.0 \pm 22.7$ & $-36.8 \pm 24.6$ & $<0.001$ \\
\hline $6 \mathrm{mo}$ & 100 & $74.1 \pm 13.9$ & $33.2 \pm 23.4$ & $-40.9 \pm 25.7$ & $<0.001$ \\
\hline $12 \mathrm{mo}$ & 88 & $74.7 \pm 13.6$ & $34.1 \pm 23.8$ & $-40.6 \pm 26.0$ & $<0.001$ \\
\hline $18 \mathrm{mo}$ & 74 & $75.5 \pm 13.4$ & $35.7 \pm 22.0$ & $-39.8 \pm 26.6$ & $<0.001$ \\
\hline $24 \mathrm{mo}$ & 68 & $76.1 \pm 12.7$ & $37.7 \pm 23.6$ & $-38.4 \pm 25.8$ & $<0.001$ \\
\hline
\end{tabular}

Abbreviations: NOSE, Nasal Obstruction Symptom Score; SD, standard deviation.

Note: Results are presented as the mean \pm SD.

${ }^{a} p$-Values are based on paired $t$-tests for change from baseline with $p<0.05$ indicating statistical significance.

As expected, the worst-case analysis results in lower NOSE responder rates and changes from baseline, especially at the 18-month and 24-month visits where there were more missing values. Assuming no change from baseline for all missing values, the NOSE responder rate is $61.1 \%$ (95\% CI $51.3 \%, 70.3 \%$ ) at 18 months and $55.0 \%$ (95\% CI $45.2 \%, 64.6 \%$ ) at 24 months. However, the mean change from baseline remained statistically significant at -27.3 at 18 months and -23.9 at 24 months (both $p<0.001$ ).

The mean reduction from baseline in nasal obstruction VAS score through 24 months is shown in - Fig. 5. The mean VAS score reduction is 29.7 points or greater and statistically significant $(p<0.001)$ at all time points.

Results of the ESS are presented in - Table 4 and - Fig. 6 . The mean baseline ESS value for the whole participant cohort is within the normal range for the ESS $(\leq 10)$, so while the changes in scores are statistically significant $(p<0.001)$, the clinical impact is unclear. However, we also examined the subgroup of participants who had baseline ESS values greater than 10 , indicating that they had problems with daytime sleepiness. These participants demonstrated mean changes that brought the postprocedure values into the normal range of less than 10, suggesting that the reduction in nasal symptoms may reduce daytime sleepiness for patients who have problems with sleepiness. The improvement was durable through 24-month follow-up.

\section{Adverse Events}

A total of 34 device-/procedure-related adverse events were reported in 26 participants ( - Table 5 ). All device/procedurerelated adverse events were mild to moderate in severity and resolved without clinical sequelae or were ongoing but stable at study completion. The most common adverse events reported included implant migration/retrieval ( $9 \%$ of participants; 10/111), pain or discomfort (4.5\% of participants; $5 / 111$ ), bumps on nose (3.6\% of participants; $4 / 111$ ), and foreign body sensation (3.6\% of participants; 4/111). Five participants underwent reimplant after device extrusion at a

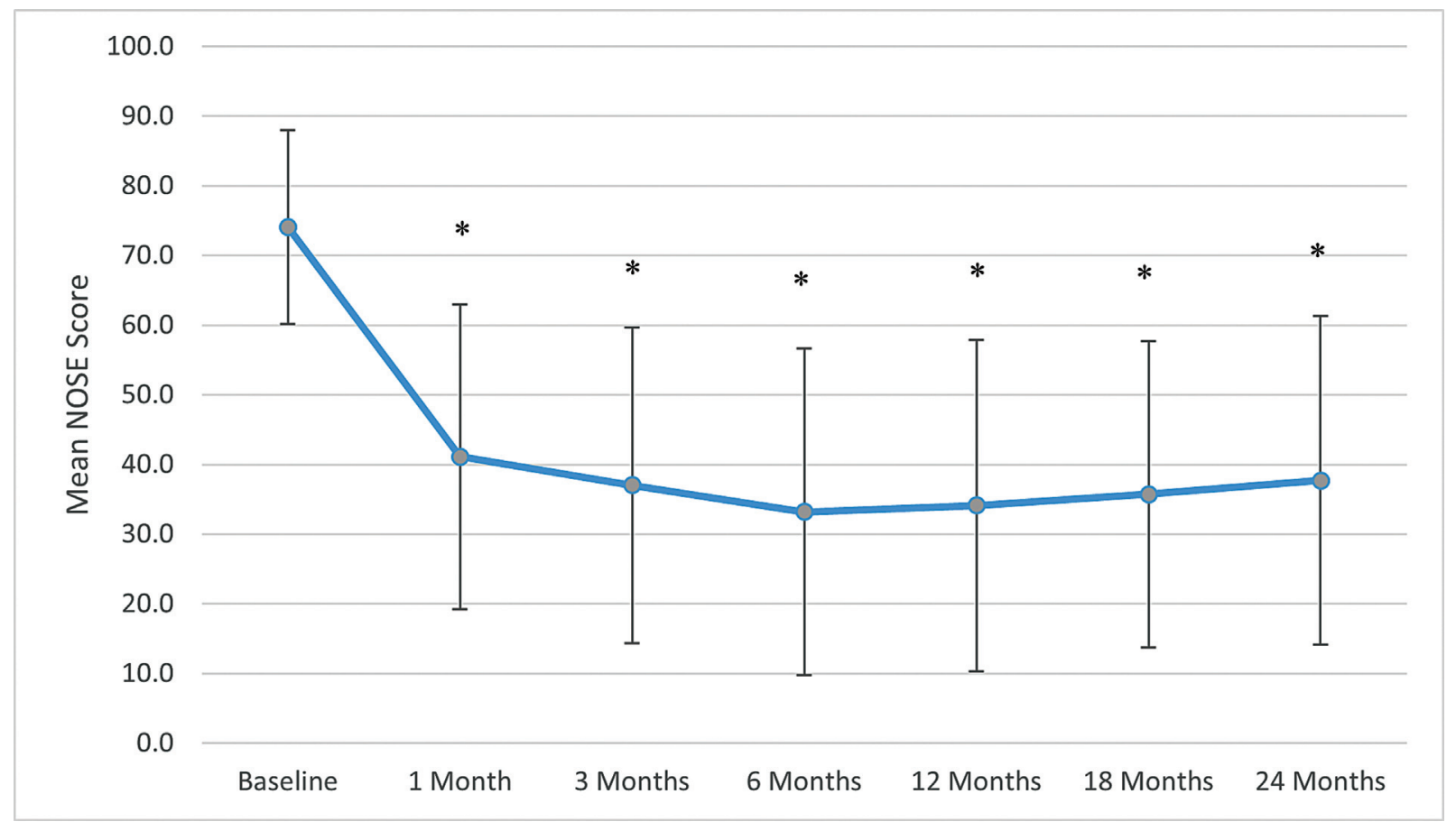

Fig. 3 Mean change in NOSE score. Error bars indicate standard deviations. Asterisk indicates $p<0.001$ compared with baseline. 


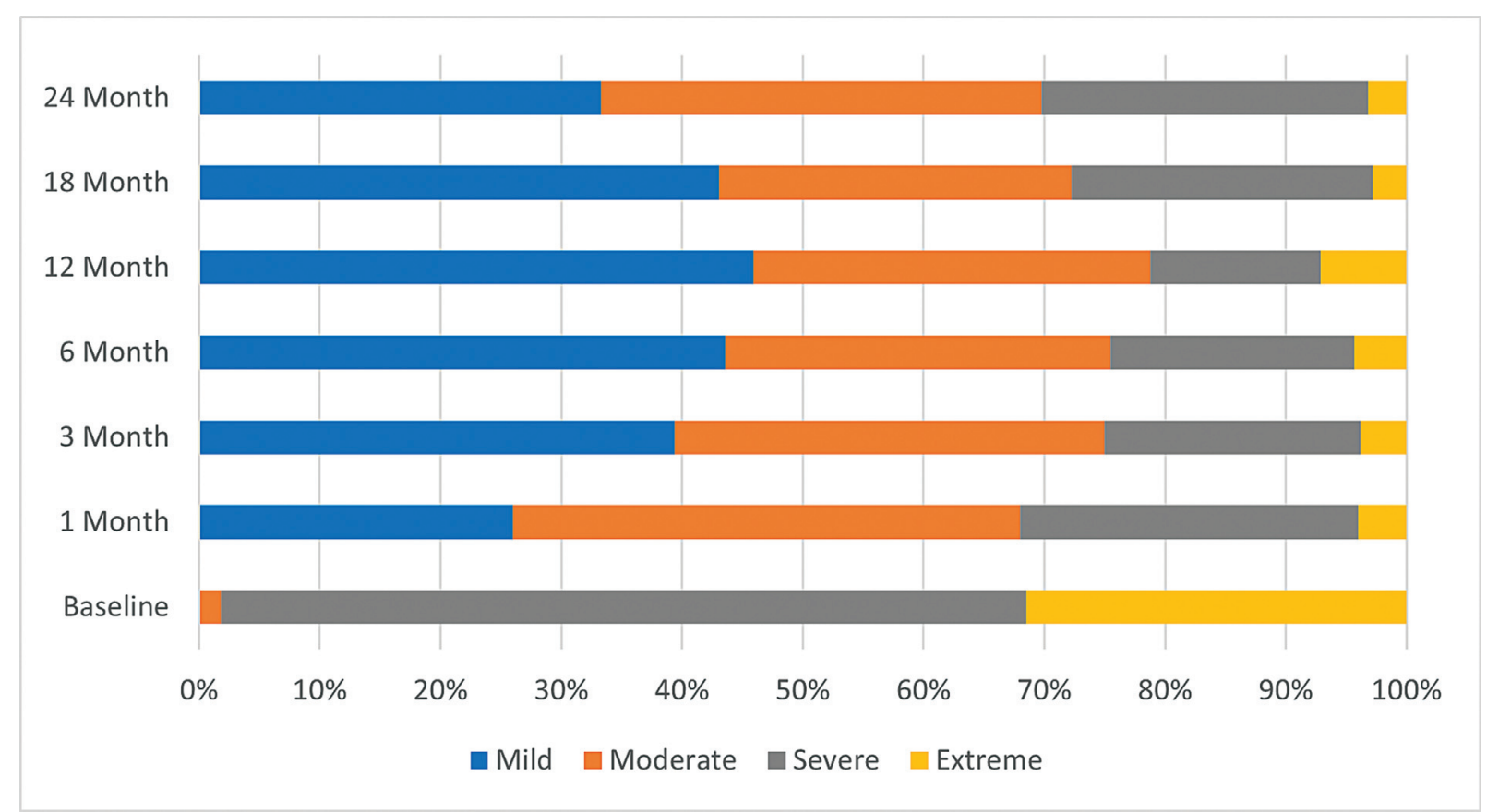

Fig. 4 Shift in NOSE severity class. NOSE scores are classified as mild (5-25), moderate (30-50), severe (55-75), extreme (80-100), as described by Lipan and Most. ${ }^{20}$

median of 21 days (range $0-133$ days) after the initial placement.

\section{Discussion}

Stolovitzky et al initially reported the 3-month randomized comparison for this randomized controlled trial. ${ }^{17}$ They demonstrated that the treatment arm was superior to the sham arm for NOSE responders ( 82.5 vs. $54.7 \%, p=0.001$ ). After the 3-month visit, sham participants could crossover to the treatment arm and complete follow-up through 24 months. Here we report the long-term follow-up of the combined treatment and crossover arms through 24 months after implant placement.

Animal histology studies have shown that the implant is absorbed over 18 to 24 months after implantation and

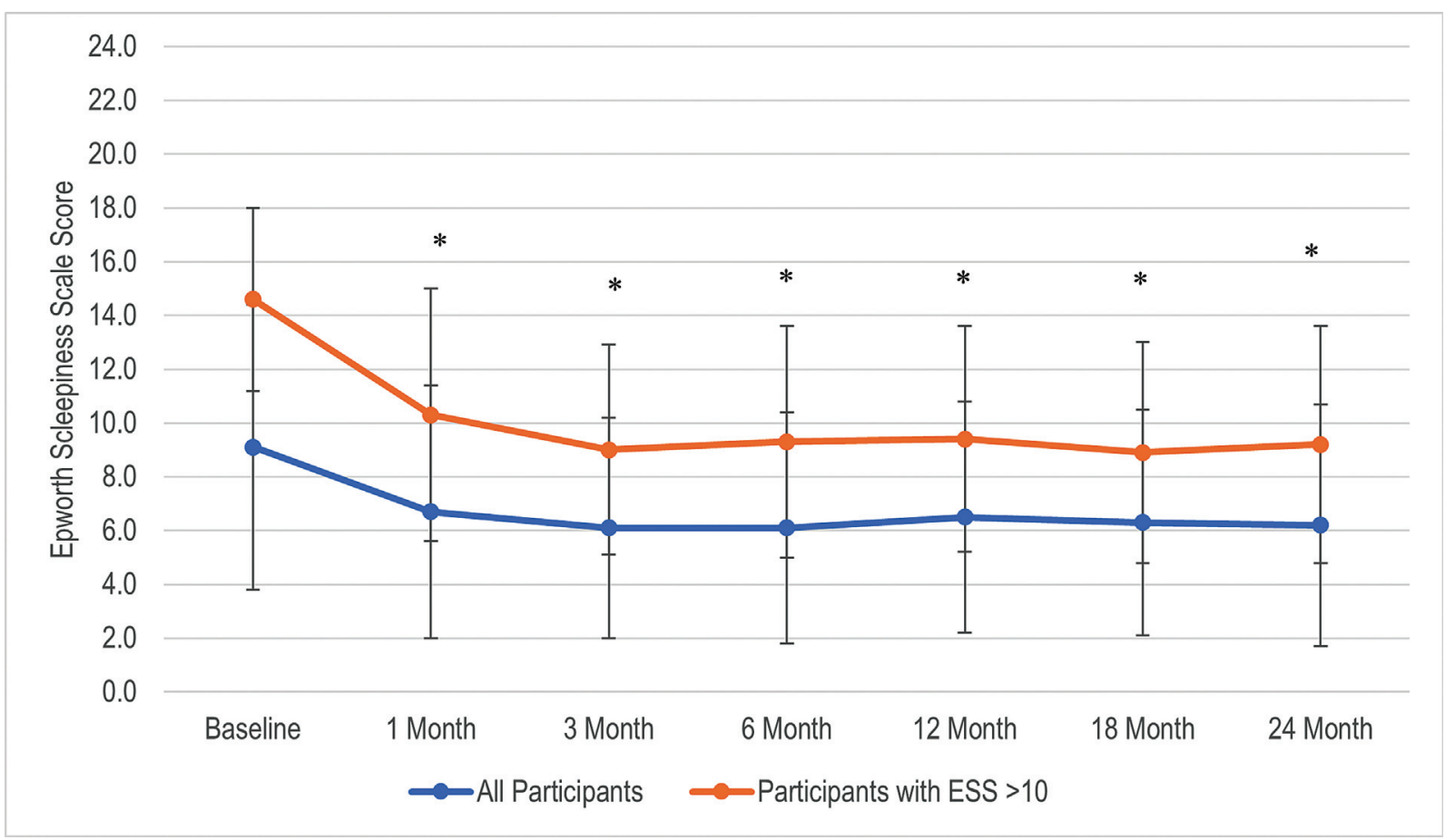

Fig. 5 Mean change in nasal obstruction VAS Score. Error bars indicate standard deviations. Asterisk indicates $p<0.001$ compared with baseline. 
Table 4 Change in mean ESS scores in all participants and in participants with abnormal baseline ESS scores

\begin{tabular}{|c|c|c|c|c|c|}
\hline Follow-up period & $N$ & Baseline ESS & Visit ESS & Mean change & $p$-Value ${ }^{a}$ \\
\hline \multicolumn{6}{|l|}{ All participants } \\
\hline $1 \mathrm{mo}$ & 104 & $9.1 \pm 5.3$ & $6.7 \pm 4.7$ & $-2.4 \pm 3.5$ & $<0.001$ \\
\hline $3 \mathrm{mo}$ & 108 & $9.0 \pm 5.1$ & $6.1 \pm 4.1$ & $-2.9 \pm 3.8$ & $<0.001$ \\
\hline $6 \mathrm{mo}$ & 100 & $9.5 \pm 5.2$ & $6.1 \pm 4.3$ & $-3.3 \pm 3.6$ & $<0.001$ \\
\hline $12 \mathrm{mo}$ & 88 & $9.4 \pm 5.2$ & $6.5 \pm 4.3$ & $-2.9 \pm 4.0$ & $<0.001$ \\
\hline $18 \mathrm{mo}$ & 74 & $9.0 \pm 5.2$ & $6.3 \pm 4.2$ & $-2.7 \pm 4.1$ & $<0.001$ \\
\hline $24 \mathrm{mo}$ & 69 & $8.7 \pm 5.2$ & $6.2 \pm 4.5$ & $-2.6 \pm 4.1$ & $<0.001$ \\
\hline \multicolumn{6}{|c|}{ Participants with abnormal baseline ESS $(>10)$} \\
\hline $1 \mathrm{mo}$ & 39 & $14.6 \pm 3.4$ & $10.3 \pm 4.7$ & $-4.2 \pm 4.1$ & $<0.001$ \\
\hline $3 \mathrm{mo}$ & 39 & $14.5 \pm 3.2$ & $9.0 \pm 3.9$ & $-5.4 \pm 4.0$ & $<0.001$ \\
\hline $6 \mathrm{mo}$ & 39 & $14.6 \pm 3.3$ & $9.3 \pm 4.3$ & $-5.3 \pm 4.1$ & $<0.001$ \\
\hline $12 \mathrm{mo}$ & 35 & $14.5 \pm 3.3$ & $9.4 \pm 4.2$ & $-5.1 \pm 4.1$ & $<0.001$ \\
\hline $18 \mathrm{mo}$ & 29 & $14.2 \pm 3.2$ & $8.9 \pm 4.1$ & $-5.3 \pm 3.9$ & $<0.001$ \\
\hline $24 \mathrm{mo}$ & 26 & $14.1 \pm 3.0$ & $9.2 \pm 4.4$ & $-4.9 \pm 4.1$ & $<0.001$ \\
\hline
\end{tabular}

Abbreviations: ESS, Epworth Sleepiness Scale; SD, standard deviation.

Note: Results are presented as the mean \pm SD.

${ }^{a} p$-Values are based on paired $t$-tests for change from baseline with $p<0.05$ indicating statistical significance.

subsequently replaced with nodular bundles of mature collagenized fibrous tissue that may provide mechanical strength at the lateral wall. ${ }^{21}$ Because the implant is resorbable, a major concern is that early results ${ }^{17}$ may diminish over time. Therefore, it was important to evaluate clinical results over at least a 24-month period.

One of the primary aims of this study was to assess the durability of the absorbable implant for NVC over 24 months. We found that NOSE scores maintained more than a 30-point reduction at all time points over the study period. Similarly, the mean VAS score reduction was more than 30 points at all time points. These results compare favorably with two other nonrandomized prospective studies using same implant. San Nicoló et al reported a mean NOSE improvement of 41 points over a 24-month period in patients with NVC. ${ }^{22}$ Similarly, Sidle et al noted a mean improvement of 44.9 points in NOSE scores and 39.5 points in VAS scores over a 24-month period. ${ }^{23}$ Our mean reductions of 36.4 points in the NOSE

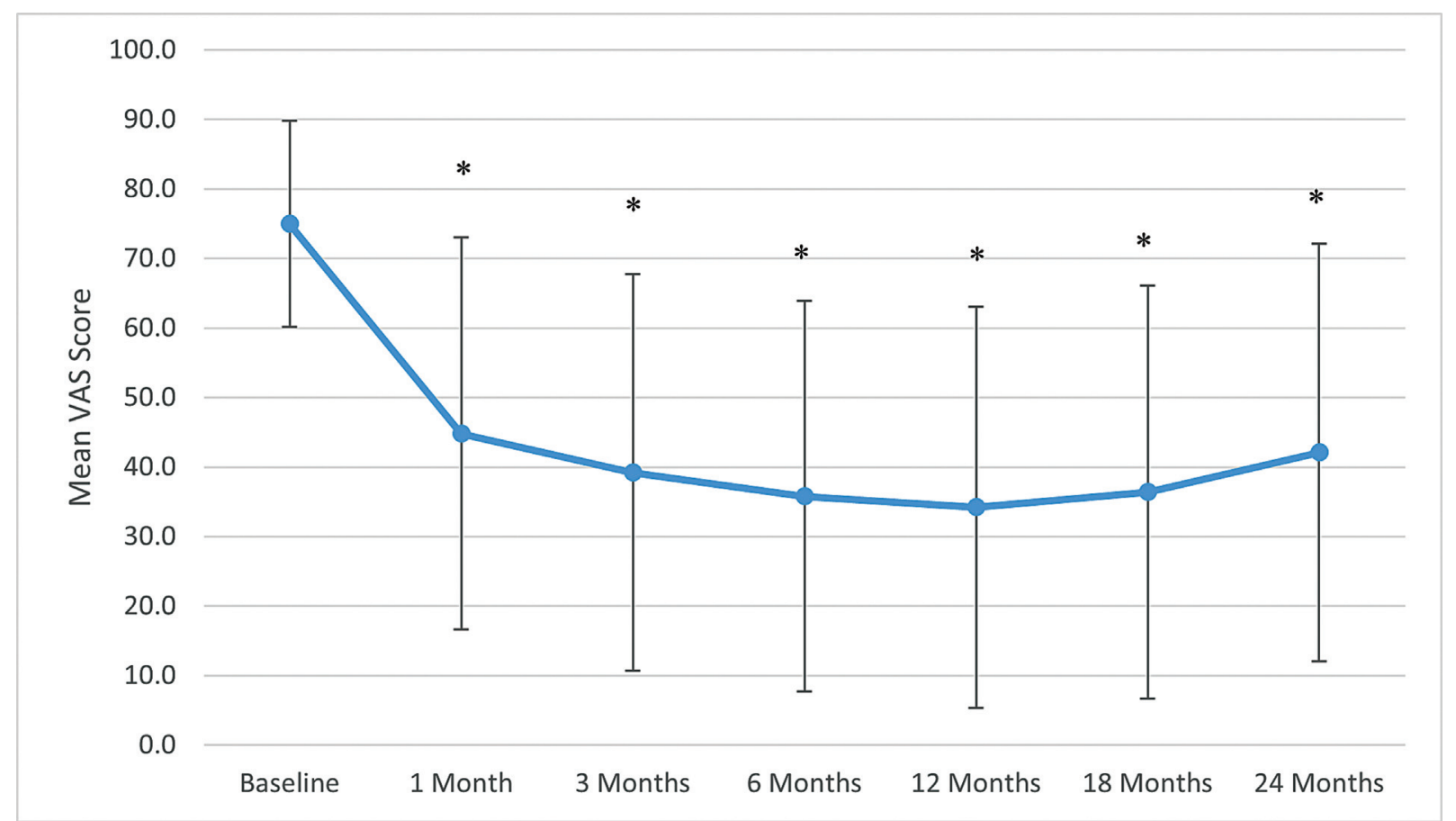

Fig. 6 Mean change in Epworth Sleepiness Scale. Error bars indicate standard deviations. Asterisk indicates $p<0.001$ compared with baseline for both groups. 
Table 5 Device-/Procedure-related adverse events

\begin{tabular}{|l|l|l|}
\hline Adverse event & Events & Participants \\
\hline $\begin{array}{l}\text { Device migration/extrusion/ } \\
\text { retrieval }\end{array}$ & 10 & 10 \\
\hline Pain or discomfort & 5 & 5 \\
\hline Bumps on nose & 4 & 4 \\
\hline Foreign body sensation & 4 & 4 \\
\hline Headache & 2 & 2 \\
\hline Inflammation & 3 & 3 \\
\hline Pin prick/pinching sensation & 2 & 2 \\
\hline Vasovagal response & 2 & 2 \\
\hline $\begin{array}{l}\text { Skin puncture with device delivery } \\
\text { needle (not implant) }\end{array}$ & 1 & 1 \\
\hline Bilateral nasal repair & 1 & 1 \\
\hline Total & 34 & $26^{\text {a }}$ \\
\hline
\end{tabular}

aThe total for participants is not the sum of the rows because a participant may have experienced more than one event.

score and 32.9 points in the VAS score at 24 months approximate the earlier studies results. In our study, both NOSE and VAS score reductions are statistically significant and consistent over 24 months, confirming durability of the procedure and implant in correcting NVC and suggesting that repeat procedures are not anticipated.

Most other invasive procedures to treat NVC are done in the operating room, requiring longer recovery time and additional costs. Our study shows that patients with dynamic NVC can benefit from a minimally invasive in-office procedure with a bioabsorbable implant. The improvement in nasal airway obstruction symptoms, measured by the mean NOSE score reduction in the treatment arm, is similar to what has been reported in surgical studies in the operating room setting. ${ }^{24-26}$

With respect to the treatment on sleep impact, it is very interesting to note that although the mean ESS is normal in the full cohort of participants, when an ad hoc analysis is done on the subgroup of participants with abnormal baseline ESS ( $>10)$, there is clinical improvement in ESS. This finding is supported by the meta-analysis by Ishii et al which demonstrated significant improvement in the ESS after isolated nasal surgery in patients with obstructive sleep apnea. ${ }^{27}$ Their pooled difference from presurgery to postsurgery ESS of 3.53 [95\% CI: $0.64,6.23$ ] was similar to the changes we observed in our study. The ESS improvement we observed suggests that this procedure indirectly has a positive clinical effect on sleep quality in those patients with sleep disturbances. This interesting finding warrants further investigation.

Most adverse device events were reported in the first few months of treatment and resolved within 6 months of the index procedure. The implant migration/retrieval rate was $4.5 \%$. The implant retrievals in this study were all unilateral, intranasal, and only one occurred later than 3 months after the initial implantation procedure. None of the retrievals were due to an adverse physiologic tissue rejection.

One strength of this study is the prospective, multicenter crossover design. The large number of treating physicians across the United States reinforces the general adaptability of the treatment in a variety of settings. The sample size of the study is also a strength with over 100 participants who underwent bilateral implantation as either the treatment or crossover arm. Finally, the use of validated patientreported outcomes, such as the NOSE, VAS, and ESS questionnaires provides validity and reproducibility to the findings.

Study limitations include the lack of long-term follow-up of the control arm, significant loss of study participants to followup at 18 and 24 months, and a lack of objective assessment of nasal valve collapse. Study participants who had received the sham procedure and continued to meet all inclusion criteria could crossover to the treatment arm at 3 months. As noted, 40 of the 66 sham participants underwent crossover to the treatment group. By study design, the 26 remaining sham participants were exited from the study after 3 months. Once the initial, 3-month randomized comparison established that the treatment arm was superior to the sham arm, the study designers/authors felt it would be unethical to continue longterm follow-up of the sham participants without allowing the remaining participants who still met inclusion criteria the opportunity to crossover to the treatment arm. While this lack of long-term follow-up of the control arm may be seen as a limitation of the study, it is unlikely that any placebo effect that was not noted during the initial 3-month interval would have presented in later months. The loss of treatment participants at 18 and 24 months, which was due in part to the coronavirus-19 pandemic of 2020 , also presented a limitation to the study. An LOCF analysis was performed to assess the impact of participant drop-out over time. To address concerns about the validity of the long-term analysis with LOCF, a worst-case scenario analysis was also performed, that validated the findings of the LOCF analysis. The current study used subjective patient assessments of nasal obstruction to assess treatment outcome effect. A more objective evaluation of nasal valve collapse, such as computed calculation of change in area of the nasal valve through endoscopic video or photo could be considered in future studies. While that type of objective analysis of nasal valve response would provide valuable data, validated patient symptom scores are useful, particularly when counseling patients on potential treatment outcome/expectations.

Finally, a notable limitation of this study is an uneven distribution of participants of varying race or ethnicity. While the enrolled population of non-White participants was low at $14 \%$, significant attempts were made in the study design to find a diverse population, such as the inclusion of 10 clinical sites across multiple geographies. While all attempts to find a diverse population were made, inherent bias toward White participants may exist. Previous studies have demonstrated racial differences in nasal anatomy that may contribute to underrepresentation of some ethnic groups. For example, Morgan et al demonstrated that Blacks have a larger minimum cross-sectional area as well as less 
nasal resistance in comparison to Caucasians, ${ }^{28}$ and thus may be less likely to need nasal surgery. In addition, to nasal anatomy variations, differences in cultural views toward surgery, or inequality in clinician attitude may influence the ethnic subsets enrolled in studies such as in this.

\section{Conclusion}

The absorbable implant is a safe and effective in-office treatment option for dynamic NVC in patients with severe to extreme nasal obstruction and provides symptom improvement through 24 months after placement.

\section{Trial Registration}

This study is registered on www.clinicaltrials.gov (NCT03400787).

\section{Conflict of Interest}

None declared.

\section{References}

1 Stewart M, Ferguson B, Fromer L. Epidemiology and burden of nasal congestion. Int J Gen Med 2010;3:37-45

2 Rhee JS, Poetker DM, Smith TL, Bustillo A, Burzynski M, Davis RE. Nasal valve surgery improves disease-specific quality of life. Laryngoscope 2005;115(03):437-440

3 Wever CC. The nasal airway: a critical review. Facial Plast Surg 2016;32(01):17-21

4 Camacho M, Riaz M, Capasso R, et al. The effect of nasal surgery on continuous positive airway pressure device use and therapeutic treatment pressures: a systematic review and meta-analysis. Sleep (Basel) 2015;38(02):279-286

5 Becker SS, Dobratz EJ, Stowell N, Barker D, Park SS. Revision septoplasty: review of sources of persistent nasal obstruction. Am J Rhinol 2008;22(04):440-444

6 Constantian MB, Clardy RB. The relative importance of septal and nasal valvular surgery in correcting airway obstruction in primary and secondary rhinoplasty. Plast Reconstr Surg 1996;98(01): 38-54, discussion 55-58

7 Ricci E, Palonta F, Preti G, et al. Role of nasal valve in the surgically corrected nasal respiratory obstruction: evaluation through rhinomanometry. Am J Rhinol 2001;15(05):307-310

8 Clark DW, Del Signore AG, Raithatha R, Senior BA. Nasal airway obstruction: prevalence and anatomic contributors. Ear Nose Throat J 2018;97(06):173-176

9 Stewart MG, Smith TL, Weaver EM, et al. Outcomes after nasal septoplasty: results from the Nasal Obstruction Septoplasty Effectiveness (NOSE) study. Otolaryngol Head Neck Surg 2004; 130(03):283-290

10 Rhee JS, Sullivan CD, Frank DO, Kimbell JS, Garcia GJM. A systematic review of patient-reported nasal obstruction scores: defining normative and symptomatic ranges in surgical patients. JAMA Facial Plast Surg 2014;16(03):219-225, quiz 232
11 Paccoi P, Di Peco V. Septal cartilage graft for nasal valve incompetence associated with deviated septum. Am J Rhinol 2007;21(05): 622-625

12 Murrell GL. Auricular cartilage grafts and nasal surgery. Laryngoscope 2004;114(12):2092-2102

13 Gunter JP, Friedman RM. Lateral crural strut graft: technique and clinical applications in rhinoplasty. Plast Reconstr Surg 1997;99 (04):943-952, discussion 953-955

14 Toriumi DM, Josen J, Weinberger M, Tardy ME Jr. Use of alar batten grafts for correction of nasal valve collapse. Arch Otolaryngol Head Neck Surg 1997;123(08):802-808

15 Islam A, Arslan N, Felek SA, Celik H, Demirci M, Oguz H. Reconstruction of the internal nasal valve: modified splay graft technique with endonasal approach. Laryngoscope 2008;118(10):1739-1743

16 Akcam T, Friedman O, Cook TA. The effect on snoring of structural nasal valve dilatation with a butterfly graft. Arch Otolaryngol Head Neck Surg 2004;130(11):1313-1318

17 Stolovitzky P, Senior B, Ow RA, Mehendale N, Bikhazi N, Sidle DM. Assessment of bioabsorbable implant treatment for nasal valve collapse compared to a sham group: a randomized control trial. Int Forum Allergy Rhinol 2019;9(08):850-856

18 Stewart MG, Witsell DL, Smith TL, Weaver EM, Yueh B, Hannley MT. Development and validation of the Nasal Obstruction Symptom Evaluation (NOSE) scale. Otolaryngol Head Neck Surg 2004; 130(02):157-163

19 Johns MW. A new method for measuring daytime sleepiness: the Epworth sleepiness scale. Sleep 1991;14(06):540-545

20 Lipan MJ, Most SP. Development of a severity classification system for subjective nasal obstruction. JAMA Facial Plast Surg 2013;15 (05):358-361

21 Rippy MK, Baron S, Rosenthal M, Senior BA. Evaluation of absorbable PLA nasal implants in an ovine model. Laryngoscope Investig Otolaryngol 2018;3(03):156-161

22 San Nicoló M, Stelter K, Sadick H, Bas M, Berghaus A. A 2-year follow-up study of an absorbable implant to treat nasal valve collapse. Facial Plast Surg 2018;34(05):545-550

23 Sidle DM, Stolovitzky P, O'Malley EM, Ow RA, Nachlas NE, Silvers S. Bioabsorbable implant for treatment of dynamic nasal valve collapse with or without concomitant procedures. Facial Plast Surg 2021;37(05):673-680

24 Chambers KJ, Horstkotte KA, Shanley K, Lindsay RW. Evaluation of improvement in nasal obstruction following nasal valve correction in patients with a history of failed septoplasty. JAMA Facial Plast Surg 2015;17(05):347-350

25 Calloway HE, Heilbronn CM, Gu JT, Pham TT, Barnes CH, Wong BJF. Functional outcomes, quantitative morphometry, and aesthetic analysis of articulated alar rim grafts in septorhinoplasty. JAMA Facial Plast Surg 2019;21(06):558-565

26 Standlee AG, Hohman MH. Evaluating the effect of spreader grafting on nasal obstruction using the NOSE scale. Ann Otol Rhinol Laryngol 2017;126(03):219-223

27 Ishii L, Roxbury C, Godoy A, Ishman S, Ishii M. Does nasal surgery improve OSA in patients with nasal obstruction and OSA? A metaanalysis. Otolaryngol Head Neck Surg 2015;153(03):326-333

28 Morgan NJ, MacGregor FB, Birchall MA, Lund VJ, Sittampalam Y. Racial differences in nasal fossa dimensions determined by acoustic rhinometry. Rhinology 1995;33(04):224-228 